Pirt, S. J. (1957). J. gen. Microbiol. 16, 59-75

\title{
The Oxygen Requirement of Growing Gultures of an Aerobacter Species Determined by means of the Continuous Gulture Technique
}

\author{
BY S. J. PIRT \\ Microbiological Research Establishment (Ministry of Supply), \\ Porton, Wiltshire
}

SUMMARY: A formula for calculating the oxygen demand of a growing culture is derived. The fate of substrate-glucose in cultures of Aerobacter cloacae was found to depend on the amount of available oxygen and the oxygen demand of the organisms. Anaerobically, cell synthesis and $\mathrm{CO}_{2}$ production are at their minimum levels and most of the glucose-carbon is converted into ethanol, formic acid, 2:3-butanediol, acetoin and acetic acid. A small supply of oxygen suppresses the formation of ethanol and formic acid but still permits the production of butanediol and acetoin and increases the proportion of glucose-carbon converted to acetic acid, cells and $\mathrm{CO}_{2}$. A larger supply of oxygen suppresses the formation of butanediol and acetoin and still further increases the yields of cells and $\mathrm{CO}_{2}$. With an excess of oxygen available, provided the growth rate of the organism is not too near its maximum value, acetic acid production is suppressed and complete conversion of the glucose-carbon into cell and $\mathrm{CO}_{2}$ occurs. At growth rates very close to the maximum a part of the glucose is converted into acetic acid even with an excess of available oxygen. The metabolism in a batch culture with excess oxygen resembles the metabolism with excess oxygen in a continuous culture in which the organisms are growing at a rate close to the maximum. Means of recognizing and preventing an oxygen deficiency are discussed.

The object of this work was to determine the effect of oxygen supply rate on the fate of glucose carbon in growing cultures of Aerobacter cloacae. It is remarkable that although carbon balances with carbohydrate as the carbon source are well defined for anaerobic 'resting' suspensions of the more commonly studied species of bacteria (e.g. see Oginsky \& Umbreit, 1954) there is no general agreement about carbon balances under aerobic conditions. Moreover, attention has been largely confined to non-growing or 'resting' organisms and consequently little is known about the carbon balances in cultures of growing organisms. Some organisms under aerobic conditions have been found to convert substrate-carbon mostly to cell-carbon and $\mathrm{CO}_{2}$ (Clifton, 1946), and Clifton has given approximate equations for the processes. Other workers, e.g. Altermatt, Simpson \& Neish (1955), found that substantial amounts of fermentation products, that is products of anaerobic metabolism, were formed from carbohydrate under aerobic conditions. Pasteur (see quotation by Stephenson, 1950) stated that when a yeast was supplied with oxygen the fate of the substrate-carbon depended on the amount of free oxygen which was available. However, no quantitative relationship between the amount of available oxygen in a yeast culture and the carbon balance was established until the work of Maxon \& Johnson (1953). The relationship between the amount of available oxygen and the carbon balance in a bacterial culture is investigated in the present paper. 


\section{THEORY}

Growth rate. For a batch culture in which logarithmic growth occurs, the law of growth is

$$
\frac{d x}{d t}=\mu x,
$$

where $x$ is the concentration of organisms, $t$ is time and $\mu$ is the growth rate constant.

If the culture volume has the constant value $V$ and fresh medium is added at a constant rate $F, F / V$ is known as the dilution rate $D$. Then in a continuous culture (Monod, 1950; Herbert, Elsworth \& Telling, 1956) we have

$$
\frac{d x}{d t}=(\mu-D) x
$$

In the steady state $\mu=D$ (since $d x / d t=0$ ).

Yield of organism. Monod (1942) showed that in cultures in which the sole growth-limiting factor was the concentration of carbon-substrate the quantity of bacteria produced was a constant fraction, $Y$, of the quantity of substrate utilized:

$$
x=Y\left(s_{0}-s\right),
$$

where $s$ is the concentration of substrate at the time $t, s_{0}$ is the initial substrate concentration and $\left(s_{0}-s\right)$ denotes the amount of substrate utilized. The constant $Y$ is called the yield constant and denotes the amount of bacteria formed from a given amount of substrate utilized.

From (3) we have

$$
\frac{d x}{d t}=\frac{-Y d s}{d t}
$$

Oxygen demand of a growing culture. Suppose that for each mole of growthlimiting substrate utilized $\boldsymbol{P}$ moles of oxygen are required. If the concentration of dissolved oxygen is $c$, the oxygen uptake $(-\Delta c)$ in time $t$, is given by $-\Delta c=P\left(s_{0}-s\right)$; hence

$$
\frac{-d c}{d t}=\frac{-P d s}{d t}
$$

where $-d c / d t$ denotes the oxygen uptake rate.

From (4) and (5) the oxygen uptake rate is directly proportional to the growth rate $\frac{-d c}{d t}=\frac{P}{\boldsymbol{Y}} \frac{d x}{d t}=\frac{\boldsymbol{P}}{\boldsymbol{Y}} \mu x$. Hence from (3)

$$
\frac{-d c}{d t}=P \mu\left(s_{0}-s\right)
$$

For a continuous culture in the steady state we may substitute $D$ for $\mu$; and generally $s$ is negligible compared with $s_{0}$. Hence (6) becomes

$$
\frac{-d c}{d t} \simeq P D s_{0}
$$


Therefore the oxygen demand of the population of organisms in a continuous culture is directly proportional to the dilution rate and to the concentration of substrate in the fresh medium. $\boldsymbol{P}$ is here called the oxygen demand constant; it may be determined by measuring the substrate utilization and the concomitant oxygen uptake.

\section{Available oxygen in cultures}

The amount of available oxygen in a submerged culture depends upon the rate of oxygen transfer from the gas to the liquid phase, that is, the oxygen solution rate $(d c / d t)$; this rate is given by the relationship

$$
\frac{d c}{d t}=\phi\left(c_{s}-c\right)
$$

where $c$ is the concentration of dissolved oxygen, $c_{s}$ is the saturation concentration of oxygen and $\phi$ is a constant which depends on the aeration conditions (Hixson \& Gaden, 1950; Bartholomew, Karow, Sfat \& Wilhelm, 1950; Chain \& Gualandi, 1954; Finn, 1954). One can readily measure the maximum possible oxygen solution rate in an aeration system by means of the sulphite oxidation method of Cooper, Fernstrom \& Miller (1944). In the sulphite solution $c$ is virtually zero, in which case $d c / d t \simeq \phi c_{s}$. In this laboratory $\phi c_{s}$ is given the symbol $\sigma$ and in this paper it will be called the oxygen absorption coefficient. The oxygen absorption coefficient $(\sigma)$ is the maximum amount of available oxygen and is expressed as mmole/l./hr. Wise (1950) reported that the polarographic method for determining oxygen absorption coefficients gave a much lower value than does the sulphite method. However, other workers (Bartholomew et al. 1950; Chain \& Gualandi, 1954) found that the two methods gave results which were in good agreement with each other. Although Wise showed that on theoretical grounds the sulphite method would be expected to give higher values than the polarographic method it is doubtful whether the theory can account for the big differences which Wise found.

Another factor on which the amount of oxygen available to organisms in submerged culture depends is the rate of oxygen transfer from the liquid to the cell. This process, it has been shown for a yeast by Winzler (1941), for some bacteria by Longmuir (1954) and for a mould by Chain \& Gualandi (1954), is independent of the concentration of dissolved oxygen unless this is very small as compared with the saturation concentration of oxygen. That being so, it follows from equation (8) that, when the oxygen uptake of the organisms is limited by the concentration of dissolved oxygen, the rate of oxygen uptake must be virtually equal to $\phi c_{s}$, the oxygen absorption coefficient $(\sigma)$. It should be noted, however, that the polarographic method requires quite small population densities of organisms to be used, probably not exceeding $0.1 \%$ dry wt., otherwise the oxygen uptake rate is too rapid to allow of accurate measurement. The conclusions drawn therefore may only be true for population densities of that order.

Rate of substrate catabolism in relation to amount of organism. The rate of substrate catabolism/unit amount of organism will be called the specific 
substrate catabolic rate. It is given the symbol $K_{c}$ when the carbon-substrate is considered. $K_{c}$ is defined by the relationship

$$
\frac{-d s}{d t}=K_{c} x
$$

From equations (4) and (1) above

$$
\frac{-d s}{d t}=\frac{\mu}{Y} x ; \text { therefore } K_{c}=\frac{\mu}{Y} .
$$

Summary of symbols and units used: $\mu=$ growth-rate constant defined by equation (1), hr. ${ }^{-1} ; \mu_{m}=$ maximum value of $\mu, \mathrm{hr}^{-1} ; D=$ dilution rate, $\mathrm{hr} .^{-1}$; $\sigma=$ oxygen absorption coefficient, mmole oxygen $/ 1$./hr.; $\boldsymbol{P}=$ oxygen demand constant, mole oxygen required/mole substrate-carbon catabolized; $\boldsymbol{Y}=$ cellyield constant $=$ organism dry wt. produced/unit quantity carbon substrate utilized (for calculating $K_{c}$ in Table 2 the units are g. organism dry wt./mmole glucose-carbon); $K_{c}=$ specific glucose catabolic rate, mmole glucose-carbon catabolized/g. dry wt. organism/hr.

\section{METHODS}

Apparatus. The continuous culture apparatus which was used and the technique of operating it were as described by Elsworth, Meakin, Pirt \& Capell (1956).

Medium A. This medium contained (amounts $/ \mathrm{l}$.) $=25 \cdot 0 \mathrm{~g} . \mathrm{KH}_{2} \mathrm{PO}_{4} ; 0.075 \mathrm{~g}$. $\mathrm{FeSO}_{4} .7 \mathrm{H}_{2} \mathrm{O} ; 0 \cdot 15 \mathrm{~g} . \mathrm{CaCl}_{2} .6 \mathrm{H}_{2} \mathrm{O} ; 0 \cdot 20 \mathrm{~g} . \mathrm{MgSO}_{4} .7 \mathrm{H}_{2} \mathrm{O} ; 0.0025 \mathrm{~g} . \mathrm{CuSO}_{4} .5 \mathrm{H}_{2} \mathrm{O}$; 0.005 g. $\mathrm{MnSO}_{4} .4 \mathrm{H}_{2} \mathrm{O} ; 0.005 \mathrm{~g}$. $\mathrm{ZnSO}_{4} .7 \mathrm{H}_{2} \mathrm{O} ; 4.0$ g. Na hexametaphosphate (Calgon). Glucose was added in different amounts; $0 \cdot 33 \mathrm{~g} .\left(\mathrm{NH}_{4}\right)_{2} \mathrm{SO}_{4}$ was added for each g. glucose. The $\mathrm{KH}_{2} \mathrm{PO}_{4}$ was adjusted to $\mathrm{pH} \mathbf{7 \cdot 3}$ with $\mathrm{KOH}$ and the $\mathrm{pH}$ of the whole medium was $7 \cdot 2-7 \cdot 3$. The phosphate, ammonium sulphate and glucose were each sterilized separately by autoclaving. The trace minerals were each dissolved separately in water and then mixed with the Calgon solution; a slight pink precipitate (presumably containing some of the manganese) was formed and removed by filtration. Since Calgon is decomposed by autoclaving, the solution of trace elements was sterilized by Seitz filtration.

Medium B. The composition was the same as that of medium A except that ethylenediamine tetracetic acid (1 g./l.) was substituted for the Calgon. The $\mathrm{pH}$ value of the chelating agent was adjusted between $\mathrm{pH} 7$ and 8 with $\mathrm{NaOH}$ before adding the mineral salts and was re-adjusted to about $\mathrm{pH} 7$ after adding the salts. The stock minerals solution with the complexing agent was sterilized by autoclaving.

Antifoam. Alkaterge C (supplied by Commercial Solvents, Terre Haute, Indiana, U.S.A.) was used for preventing foaming. It was diluted with medicinal paraffin to a strength of $30 \%(\mathrm{v} / \mathrm{v})$. Two or three drops of this preparation were added by means of the antifoam meter as soon as the foam formed a continuous layer over the liquid. The concentration of the antifoam preparation was thus maintained near $0.01 \%$ (v/v.).

Temperature. The temperature of the culture was maintained at $37^{\circ} \pm 0 \cdot 5^{\circ}$.

Seed cultures. The organism used was Aerobacter cloacae strain NCTC 8197. 
(In a preliminary account of this work (Pirt, 1956) the organism was called Aerobacter aerogenes.) The inoculum for the reactor consisted of 100-200 ml. of culture produced in shaken flasks. The seed flask was inoculated from a stock culture of the organism on tryptic digest of meat agar. The organism was adapted to the defined medium in two stages. In the first seed flask the concentrations of all salts were decreased to about half strength; in the second seed the concentration of the salts was full strength. In both inocula the glucose concentration was $1 \mathrm{~g}$./ 1 . The oxygen absorption coefficient of the inocula was about $45 \mathrm{mmole} / \mathrm{l} / \mathrm{hr}$.

Preparation and preservation of samples. The work required many different analyses to be carried out on the same sample and since they could not be done simultaneously a method of preserving samples was essential. Refrigeration at a temperature just above freezing-point was not adequate. The method adopted was to add one drop of a saturated solution of mercuric chloride to each $50 \mathrm{ml}$. of sample. This treatment killed all organisms in $0.4 \%$ suspensions and prevented further growth in the cell-free liquor. After removing organisms by centrifugation the samples were stored in the refrigerator $\left(3^{\circ}\right)$. The mercuric chloride did not interfere in any of the analytical techniques; the amount of it was too small to remove any appreciable amount of formic acid.

Dry weight of organisms. The dry weight of organisms used was determined directly by weighing, except for a few cases in which it was calculated from the optical density. A sample containing about $50 \mathrm{mg}$. dry wt. organisms was centrifuged at $15,000 \mathrm{~g}$. for about $5 \mathrm{~min}$. to separate organisms from medium. The organisms were washed twice by re-suspending in water. The washed organisms, re-suspended in a little water, were then transferred by a Pasteur pipette to a weighed Pyrex evaporating dish. The suspension was evaporated to dryness on a water bath and then dried to constant weight in an oven at $100^{\circ}-105^{\circ}$. In calculating dry weights from optical densities it was necessary to know the ratio optical density:dry wt. at the particular growth rate which the organisms had, since it has been found, in this laboratory, that this ratio is a function of the growth rate of the organisms.

Cell carbon. Carbon analyses by dry combustion were carried out on samples of organisms grown anaerobically and with excess oxygen. The proportion of carbon in the sample of organisms grown anaerobically was $49.6 \%$; in that aerobically grown, $50 \cdot 8 \%$. The mean of these two values $(50 \cdot 2 \%)$ was used to calculate the amounts of cell-carbon from the dry weight of organisms.

Carbon dioxide. The total $\mathrm{CO}_{2}$ evolved by the culture is the sum of the $\mathrm{CO}_{2}$ in the effluent gas and that retained by the medium. The percentage of $\mathrm{CO}_{2}$ in the effluent gas from the fermentation vessel was determined by means of an Orsat gas-analysis apparatus. The $\mathrm{CO}_{2}$ retained by the culture medium was determined by the microdiffusion method of Conway (1947); a sample of culture for the purpose was taken anaerobically. This was done by collecting the sample under a layer of liquid paraffin as recommended for blood analysis by Conway. The $\mathrm{CO}_{2}$ concentration found slightly exceeded the theoretical value calculated by assuming that the dissolved $\mathrm{CO}_{2}$ was in equilibrium with the $\mathrm{CO}_{2}$ in the effluent gas. 
Total volatile acid. The sample ( $4 \mathrm{ml}$.) was acidified with $10 \mathrm{~N}-\mathrm{H}_{2} \mathrm{SO}_{4}(0 \cdot 2 \mathrm{ml}$.) and steam distilled. Formic acid and acetic acid recoveries were complete after $50 \mathrm{ml}$. (that is, about $12 \mathrm{vol}$.) had distilled over. The distilled acid was titrated with $\mathrm{CO}_{2}$-free $0.01 \mathrm{~N}-\mathrm{NaOH}$, using phenol red as indicator. Sharp end points and very small blank values were obtained without bubbling $\mathrm{CO}_{2}$-free nitrogen through the solution to be titrated, provided that it was freshly distilled.

Formic acid and acetic acid. That part of the volatile acid which was destroyed by mercuric salts was taken to be formic acid. A technique for obtaining complete destruction of formic acid was worked out. $\mathrm{HgO}(310 \pm 10 \mathrm{mg}$.) was placed in a $10 \mathrm{ml}$. flask, the sample $(5 \mathrm{ml}$.) was added, followed by conc. $\mathrm{H}_{2} \mathrm{SO}_{4}(0 \cdot 2 \mathrm{ml}$.), and the contents of the flask were gently boiled under reflux on a sand bath for $20 \mathrm{~min}$. This amount of $\mathrm{HgO}$ was more than sufficient to destroy $20 \mathrm{mg}$. formic acid. On distillation of a portion $(4 \mathrm{ml}$.) of the residual solution, acetic acid was completely recovered and estimated by titration. Acetic acid was identified by the lanthanum nitrate spot test.

Ethanol. The microdiffusion technique of Conway (1947) was used for the determination of ethanol. A correction was made for the small amount of acetoin which diffused with the ethanol.

Acetoin. This substance was estimated colorimetrically by the $\alpha$-naphthol method described by Neish (1952) with the difference that $1 \% \alpha$-naphthol was used; this modification decreased the blank value.

2:3-Butanediol. Butanediol was oxidized with bromine water as described by Happold \& Spencer (1952). After the reduction of excess bromine with ferrous sulphate, $10 \mathrm{ml}$. of the solution were half-distilled without fractionation (Neish, 1952). The oxidation products in $4 \mathrm{ml}$. distillate were estimated by the colorimetric method used for acetoin. Acetoin was subjected to the same procedure to determine the correction to be made for it.

Glucose. The method of ferricyanide reduction was used for the determination of glucose. The reagent was that of Fujita \& Iwatake (1931) with increases in the concentrations of constituents to allow a sample of 2-3 mg. glucose to be taken.

Aeration. Oxygen absorption rates were measured by the sulphite method (Cooper et al. 1944). The oxygen absorption coefficients found for the various aeration conditions used are given in Table 1.

Table 1. Oxygen absorption coefficients $(\sigma)$ and aeration conditions

\begin{tabular}{|c|c|c|c|}
\hline \multirow[b]{2}{*}{ mmole $\stackrel{\sigma}{\mathrm{O}_{2} / 1 . / \mathrm{hr}}$} & \multicolumn{2}{|c|}{ Impeller } & \multirow[b]{2}{*}{$\begin{array}{c}\text { Air flow } \\
\text { (1./l. culture/min.) }\end{array}$} \\
\hline & $\begin{array}{l}\text { diameter } \\
(\mathrm{mm} .)\end{array}$ & r.p.m. & \\
\hline 463 & 73 & 1030 & $\mathbf{2} \cdot \mathbf{0}$ \\
\hline 228 & 63 & 1030 & $1 \cdot 0$ \\
\hline 85 & 63 & 1030 & $0 \cdot 2$ \\
\hline 24 & 63 & 715 & 0.2 \\
\hline
\end{tabular}

Note. The air pressure in the reactor was about 6 in. water gauge above atmospheric pressure. 
Anaerobic conditions. Anaerobic conditions were obtained by maintaining an atmosphere of nitrogen in the whole apparatus; 0.2 vol. of cylinder nitrogen/vol. culture was sparged through the reactor with stirring at 715 r.p.m. Nitrogen was also bubbled through the medium reservoirs.

Evaporation. Evaporation of the culture was neglected since it was only about $3 \mathrm{ml} . / \mathrm{l} . / \mathrm{hr}$. at $37^{\circ}$ with the most intense aeration $(\sigma=463)$ used.

\section{RESULTS}

The $\mathrm{pH}$ value of cultures. The $\mathrm{pH}$ value of continuous cultures in the steady state was $6 \cdot 7 \pm 0 \cdot 2$ with $10 \cdot 6 \mathrm{~g}$. glucose/l. in the fresh medium. With $19 \cdot 9 \mathrm{~g}$. glucose/l. the $\mathrm{pH}$ value was $6 \cdot 3 \pm 0 \cdot 2$. In the batch cultures the $\mathrm{pH}$ value fell steadily from the initial value $7 \cdot 3$ to $6 \cdot 6$. Automatic $\mathrm{pH}$-control apparatus was not used in this work.

Glucose utilization and maximum cell growth rates at various oxygen supply rates

At both glucose levels used (10.6 and $19.9 \mathrm{~g}$./l.) the addition of more of the medium constituents other than glucose did not affect the population density; growth was limited by the amount of glucose present. The utilization of glucose

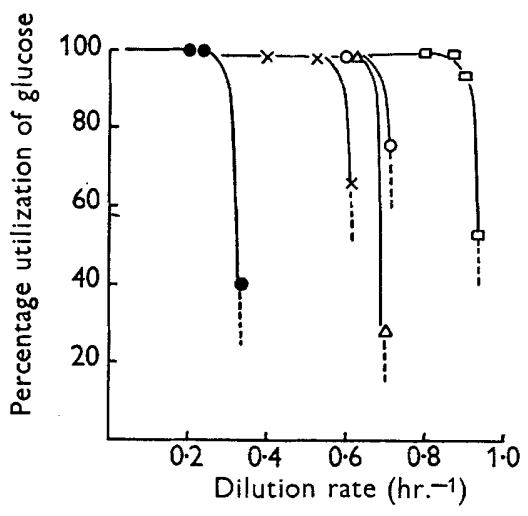

Fig. 1

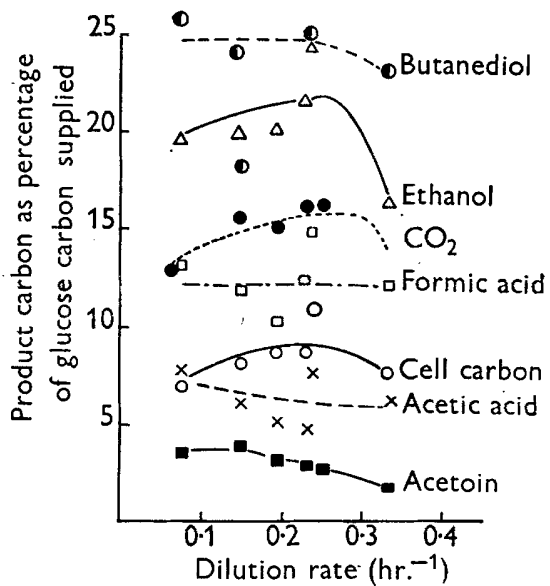

Fig. 2

Fig. 1. Glucose utilization at different dilution rates. Glucose supplied, 10.6 g./I. Oxygen absorption coefficients in mmole/l./hr.: $\square, 228$ or 463 (medium $A$ ); 0,228 (medium B); $\triangle, 85$ (medium B); $\times, 24$ (medium B); 0 , 0 (medium B).

Fig. 2. End products of anaerobic glucose metabolism as a function of dilution rate in continuous culture with medium B. Glucose supplied, $10 \cdot 6 \mathrm{~g} . / 1$.

at different dilution rates with various oxygen absorption coefficients is depicted in Fig. 1. The glucose utilization was virtually complete as long as the dilution rate was not nearly equal to the maximum growth-rate constant $\left(\mu_{m}\right)$. The dilution rate at which the glucose utilization decreased sharply was a rough measure of $\mu_{m}$. It can be seen from Fig. 1 that medium A permitted a higher 
growth rate than did medium B. Also from the results with medium B (Fig. 1) it is apparent that when the oxygen absorption coefficient fell below a certain value (between 85 and 228) the maximum growth rate also decreased.

\section{Anaerobic metabolism}

When the organism was grown anaerobically the glucose-carbon was accounted for as organisms, $\mathrm{CO}_{2}$, acetic acid, formic acid, ethanol, butanediol and acetoin. The average amount of carbon accounted for as known products was $92 \%$, with a standard deviation of 8 . The graphs in Fig. 2 show the percentage of substrate-carbon converted to the various products at different dilution rates. The percentage of total carbon which appeared in the organisms reached a maximum of 9 at a dilution rate of $0 \cdot 25$. At the same dilution rate the percentage of glucose-carbon converted to $\mathrm{CO}_{2}$ also reached the maximum value of $16 \%$. The amount of formic acid produced at dilution rates almost up to $\mu_{m}$ was fairly constant, corresponding to about $12 \%$ of the total carbon. About $6 \%$ of the total carbon was converted to acetic acid. Ethanol production under anaerobic conditions accounted for about $22 \%$ of the total glucosecarbon utilized. The slight decrease in ethanol yields at lower dilution rates is attributed to evaporation of ethanol; the decrease in the concentration is roughly what one would expect on the basis of data for ethanol evaporation given by Maxon \& Johnson (1953). The butanediol production accounted for about $24 \%$ of the carbon supplied and was uninfluenced by the dilution rate until it was close to $\mu_{m}$. Acetoin production was relatively small and increased slightly at the lower dilution rates. The sharp decrease in the yields of products at the highest dilution rates was due to the rapid fall in sugar utilization which occurred when the growth rate constant was near its maximum value. The main conclusion to be drawn from the study of the anaerobic metabolism is that it is largely independent of the dilution rate and consequently of the growth rate until, of course, the growth rate is sufficiently near to its maximum value to cause the glucose utilization to be appreciably incomplete.

\section{Fully aerobic metabolism}

The metabolism of glucose at $10 \cdot 6 \mathrm{~g}$./1. with an oxygen absorption coefficient of $228 \mathrm{mmole} / \mathrm{l} / \mathrm{hr}$. may next be considered. The average amount of carbon accounted for was $93 \%$; standard deviation 5. The results are shown in Fig. $3 a$ and $b$. The amount of glucose-carbon converted into cell-carbon reached a maximum corresponding to $55 \%$ of the glucose-carbon supplied, at a dilution rate of $0 \cdot 6$. It was shown with medium $B$ that at dilution rates up to $\mathbf{0 . 6}$ practically all of the substrate carbon could be accounted for as organisms and $\mathrm{CO}_{2}$. At growth rates near the maximum an appreciable amount of acetic acid was produced. Apart from these products small amounts of ethanol and formic acid (0-3\% of the total carbon, see Fig. 4) were found at the higher flow rates. The yields of organism and $\mathrm{CO}_{2}$ were, respectively, about 5 and 2.5 times those obtained under anaerobic conditions. At the lower dilution rates lower yields of organism were found and the yield of $\mathrm{CO}_{2}$ tended to 
increase. There were two differences between the results obtained with medium A and medium B. The maximum growth-rate constant was higher with medium A, presumably due to the difference in mineral balance. Secondly, volatile acid production started at a higher growth-rate constant with medium $A$ than with medium B; but in each case the growth-rate constant at which volatile acid production started was close to $0 \cdot 85 \mu_{m}$.

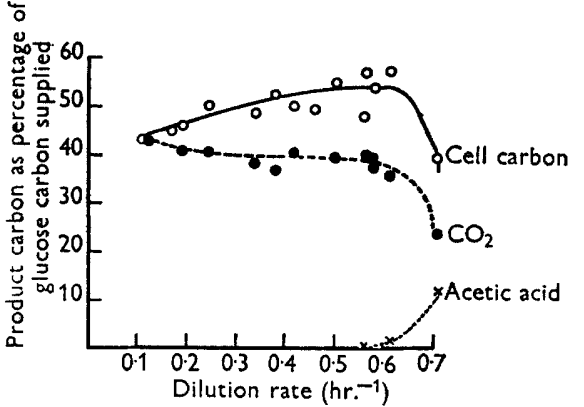

$\boldsymbol{a}$

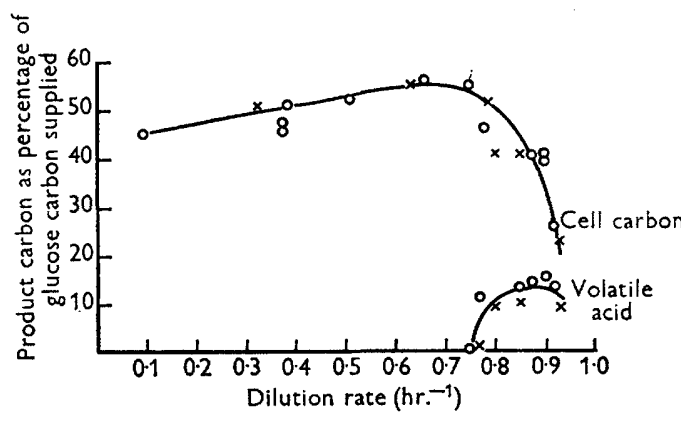

b

Fig. 3a. End products of glucose metabolism as a function of dilution rate with medium $B$ and an oxygen absorption coefficient of $228 \mathrm{mmole} / \mathrm{l} . \mathrm{hr}$. Glucose supplied, $10 \cdot 6 \mathrm{~g} . / 1$.

Fig. $3 b$. End products of glucose metabolism as a function of dilution rate with Medium $\mathbf{A}$. Oxygen absorption coefficients (mmole/l./hr.): $\bigcirc, 228 ; \times, 463$. Glucose supplied, $10 \cdot 6 \mathrm{~g} . / \mathrm{l}$. Volatile acid estimated as acetate. Acetic acid and formic acid were not separately determined in experiments with medium $\mathbf{A}$.

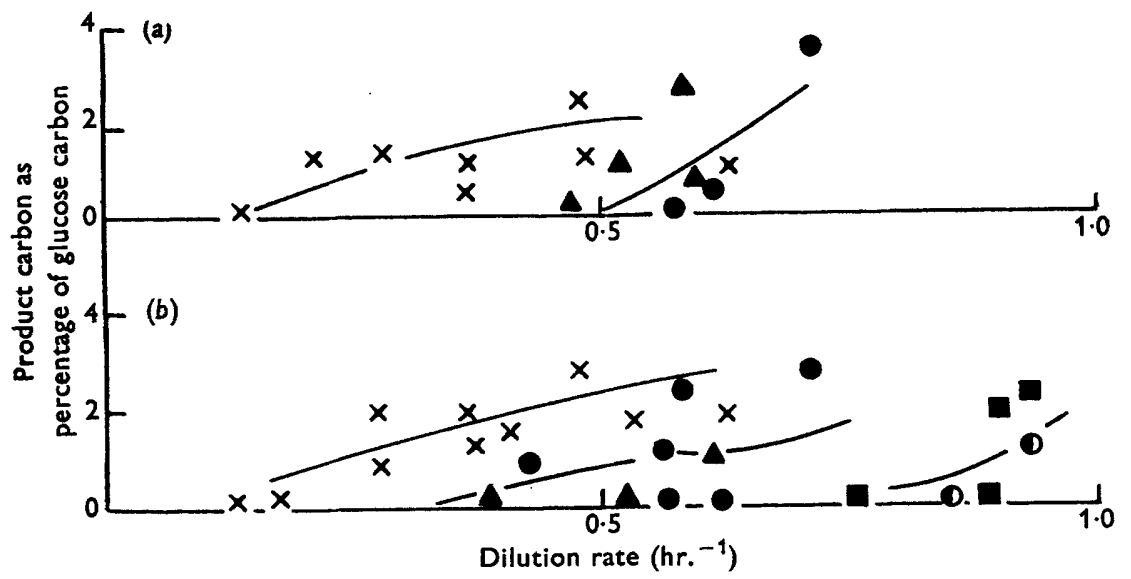

Fig. 4. Ethanol (a) and formic acid production (b) as a function of dilution rate in aerobic cultures. Oxygen absorption coefficients in mmole/1./hr. with medium B: $\mathbf{0}, 228$; $\Delta, 85 ; \times, 24$. With medium $A: \square, 228 ; 0,463$. Glucose supplied, $10 \cdot 6 \mathrm{~g} . / 1$.

When the oxygen absorption coefficient was increased from 228 to $463 \mathrm{mmole} / \mathrm{l}$./hr. with medium A (see Fig. $3 b$ ) the yield of organisms was unchanged and volatile acid production started at the same dilution rate with either degree of aeration. The slightly lower peak conversion of glucose into 
volatile acid with the higher oxygen absorption coefficient is probably due to some volatilization of the acid as a result of the intense aeration. Since the increase in the oxygen absorption coefficient caused no apparent change in the end products of glucose metabolism it seems probable that an excess of oxygen was available. We can also decide whether there was an excess of available oxygen by calculating the maximum oxygen demand of the culture by means of equation (7), and comparing it with the maximum amount of available oxygen, that is, the oxygen absorption coefficient. From the results, an approximate value of $\boldsymbol{P}$ for fully aerobic metabolism may be obtained by assuming that the R.Q. is unity and hence equating oxygen uptake with $\mathrm{CO}_{2}$ production. From the data of Fig. $3 a$ therefore it is concluded that approximately 0.4 mole oxygen is required for each mole of substrate-carbon metabolized. Thus $\boldsymbol{P}$, the oxygen demand constant, is approximately $\mathbf{0 \cdot 4}$ for fully aerobic metabolism. The value of $\boldsymbol{P}$ for growing a yeast under fully aerobic conditions which one can calculate from the data of Maxon \& Johnson (1953) is also about $\mathbf{0} \cdot 4$. The calculated maximum oxygen demand of the culture with medium $B$ and 10.6 g. glucose (354 mmole carbon) $/$ l. occurs at a dilution rate of $0.7 \mathrm{hr}^{-1}$ and is $99 \mathrm{mmole} / \mathrm{l} . / \mathrm{hr}$. But since the oxygen absorption coefficient was $228 \mathrm{mmole} / \mathrm{l}$./hr. there should have been a large excess of available oxygen.

\section{Partially aerobic metabolism}

In order to achieve partially aerobic metabolism with medium $\mathbf{B}$ the oxygen absorption coefficient was made less than the theoretical maximum oxygen demand of $99 \mathrm{mmole} / \mathrm{l} / \mathrm{hr}$. First, an oxygen absorption coefficient of $85 \mathrm{mmole} / \mathrm{l}$./hr. was used and secondly an oxygen absorption coefficient of $24 \mathrm{mmole} / \mathrm{l}$./hr. With these oxygen absorption coefficients one calculates from equation (7), taking $P=0 \cdot 4$, that the oxygen solution rate should become a limiting factor at dilution rates of $\mathbf{0 \cdot 6}$ and $\mathbf{0 \cdot 2}$, respectively.

The results obtained using an oxygen absorption coefficient of $85 \mathrm{mmole} / \mathrm{l} . / \mathrm{hr}$. are shown in Fig. 5. With dilution rates less than $\mathbf{0 . 4 5}$ the metabolism was the same as that with an oxygen absorption coefficient of 228. It may be concluded, therefore, that the metabolism was fully aerobic at dilution rates up to $\mathbf{0 \cdot 4 5}$. At higher dilution rates the yields of organism and $\mathrm{CO}_{2}$ fell below those found with an oxygen absorption coefficient of 228. Also, at a dilution rate of 0.45 acetic acid production started, whereas with an oxygen absorption coefficient of 228 acetic acid production did not occur until the dilution rate exceeded 0.6. Acetoin or butanediol were not formed until the dilution rate reached $\mathbf{0 . 6}$ when traces (about $0.5 \%$ of the total carbon) were detected. Traces of formic acid and ethanol were produced as the graphs in Fig. 4 show. The average amount of glucose-carbon accounted for was $90 \%$; standard deviation 5. The dilution rate of $\mathbf{0 . 4 5}$ at which the carbon balance began to diverge from that characteristic of fully aerobic metabolism is in reasonably good agreement with the calculated dilution rate $(0 \cdot 6)$ at which the oxygen solution rate should become a limiting factor.

The graphs showing the results obtained with an oxygen absorption coeffi- 
cient of $24 \mathrm{mmole} / \mathrm{l} . / \mathrm{hr}$. are given in Fig. 6. The average amount of carbon accounted for was $87 \%$; standard deviation 6 . Under these conditions, at dilution rates up to $\mathbf{0} \cdot \mathbf{2}$, the end products found (i.e. organisms and $\mathrm{CO}_{2}$ only) were the same as with higher oxygen absorption coefficients. The metabolism

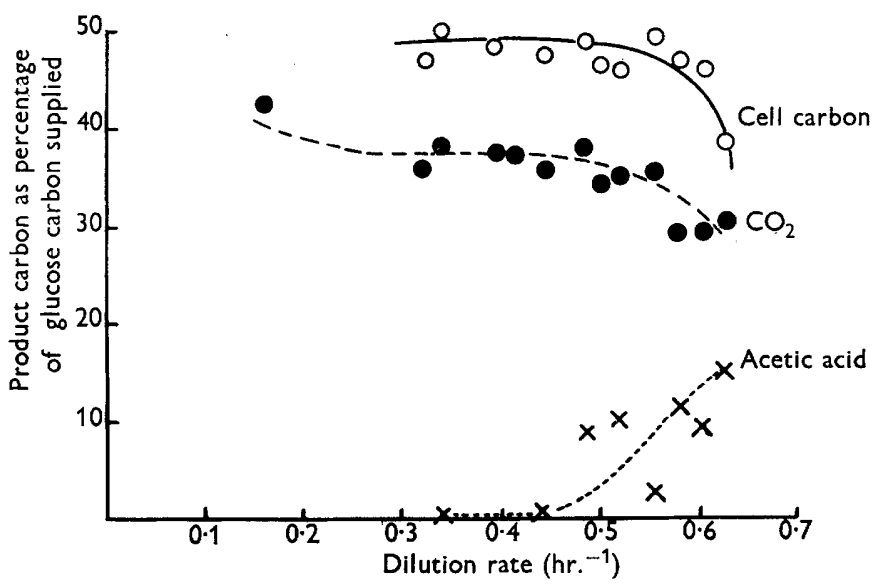

Fig. 5. End products of glucose metabolism as a function of dilution rate with an oxygen absorption coefficient of $85 \mathrm{mmole} / \mathrm{l} . / \mathrm{hr}$. Medium B; glucose supplied, $10 \cdot 6 \mathrm{~g} . / \mathrm{l}$.

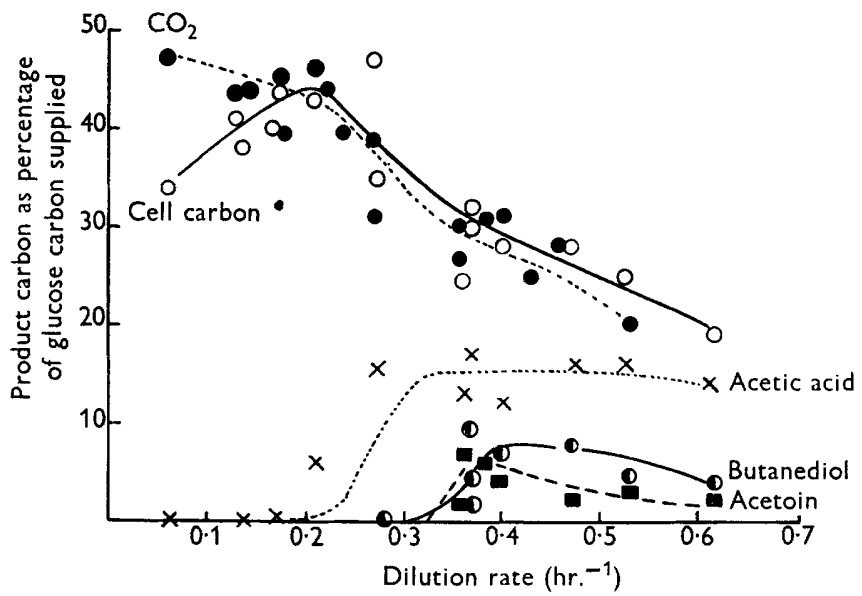

Fig. 6. End products of glucose metabolism as a function of dilution rate with an oxygen absorption coefficient of $24 \mathrm{mmole} / \mathrm{l} / \mathrm{hr}$. Medium B; glucose supplied, $10 \cdot 6 \mathrm{~g} . /$.

must therefore be fully aerobic at dilution rates up to $0 \cdot 2$, which is the same value as the calculated dilution rate at which the oxygen solution rate should become limiting. When the dilution rate exceeded $0 \cdot 2$ acetic acid was the first new product to appear. Acetic acid production reached its maximum at a dilution rate of $\mathbf{0} \cdot \mathbf{3}$. At this dilution rate (i.e. when acetic acid production reached its peak) acetoin and butanediol production began. Formic acid and ethanol were produced in small quantities (1-3\% of the total carbon) at the 
higher dilution rates (Fig. 4). Apparently formic acid and ethanol were formed in appreciable amounts only under almost anaerobic conditions. The fact that aeration suppressed ethanol formation but permitted butanediol production was also observed in studies of 2:3-butanediol production by Aerobacter aerogenes (Ledingham \& Neish, 1954). It is concluded therefore that in partially aerobic metabolism the nature of the end products of carbon metabolism depends on the oxygen deficiency, which is defined as the difference between the amount of oxygen available and the amount required for fully aerobic growth. A small oxygen deficiency will lead to the formation of acetic acid, a larger one causes butanediol and acetoin production; and with no oxygen available formic acid and ethanol production occur and acetic acid production is decreased. Presumably the oxygen deficiency exerts its effect by governing the concentration of dissolved oxygen. The $\mathrm{pH}$ value also affects the ratio of the amounts of different products formed with partially aerobic metabolism.

\section{Tests for other metabolic products}

Besides those substances which this strain of Aerobacter cloacae produces a number of other likely products were tested for but found to be absent. They were: pyruvic acid in partially aerobic and anaerobic metabolism; diacetyl in partially aerobic metabolism; lactic acid with fully aerobic, partially aerobic and anaerobic metabolism; succinic acid with fully aerobic metabolism. Hydrogen, which may be produced by the dehydrogenation of formic acid, was not tested for. Some determinations of the total non-volatile carbon in the cultures (by dry combustion) revealed that $97-100 \%$ was accounted for as known products; hence most of the glucose-carbon not accounted for as known products was probably in the volatile fraction.

\section{Metabolism in batch culture}

The growth-rate constants of batch cultures started from seed cultures varied from one culture to another and generally were lower than the maximum growth-rate constant always obtained in continuous cultures. Presumably this was because better adaptation to the new. medium occurred when the culture was prolonged. The formation of metabolic products during growth of the organism in batch culture with medium B is depicted in Fig. 7. The glucose concentration was $c .10 \mathrm{~g} . / \mathrm{l}$. and the oxygen absorption coefficient $228 \mathrm{mmole} / \mathrm{l}$. $/ \mathrm{hr}$. The $\mathrm{CO}_{2}$ produced was not measured but probably accounted for at least $30 \%$ of the total carbon (from measurements of $\mathrm{CO}_{2}$ production in continuous cultures with the growth-rate constant very close to its maximum value). Assuming a value of $30 \%$ for the $\mathrm{CO}_{2}$ production the carbon recovery would be $91 \%$. The results show that acetic acid was produced throughout the batch culture and accounted for $15 \%$ of the glucose-carbon utilized. A small amount of formic acid was produced towards the end of the culture but it was metabolized after the glucose was exhausted. No appreciable amounts of ethanol, acetoin or butanediol were detected. In another batch culture with a higher oxygen absorption coefficient ( $463 \mathrm{mmole} / \mathrm{l} . / \mathrm{hr}$.) and using medium $\mathrm{A}$ 
the growth-rate constant was higher but organisms and acetic acid were produced in the same proportions as the data in Fig. 7 indicate. The cell-yield constant $(Y)$ in the batch cultures was $0.32 \mathrm{~g}$. dry wt. organisms/g. glucose, which is much lower than the maximum $(0 \cdot 44)$ obtained in a continuous culture with a dilution rate of $\mathbf{0 . 6}$ and an oxygen absorption coefficient of 228 , but about the same as the yield obtained in a continuous culture operating at the highest growth rate. The possibility that the acetic acid was metabolized

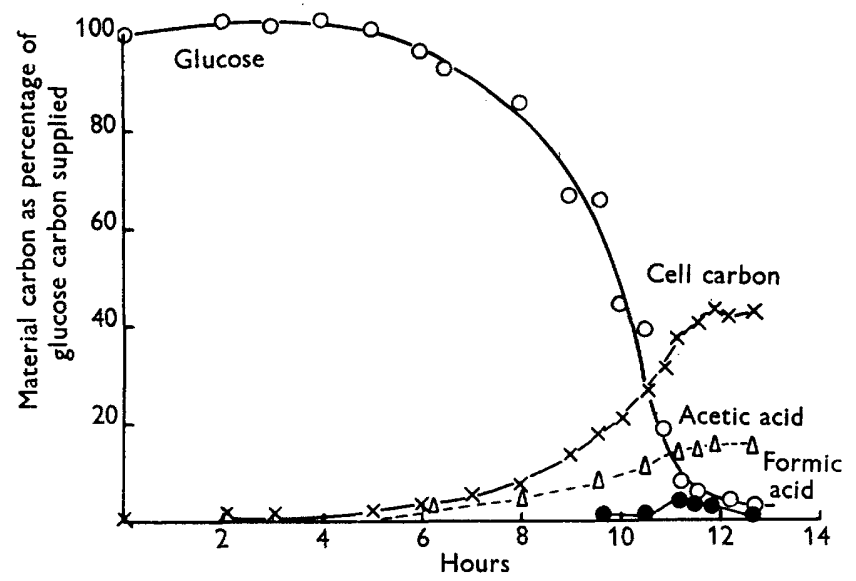

Fig. 7. Products of glucose metabolism in batch culture as a function of time. Medium B; glucose supplied, 9.6 g./1.; oxygen absorption coefficient $228 \mathrm{mmole} / \mathrm{l} . / \mathrm{hr}$.

later in the batch culture after exhaustion of the glucose was not investigated. Thus the metabolism in these batch cultures seems the same as fully aerobic metabolism in a continuous culture with the growth rate of the organism very close to its maximum value. In view of the decreased $\mathrm{CO}_{2}$ yields at growth rates close to the maximum, the value of the oxygen demand constant in batch cultures probably was less than $\mathbf{0} \cdot \mathbf{4}$, however, using the value $\mathbf{0} \cdot \mathbf{4}$ for $\boldsymbol{P}$ one calculates by means of equation (6) that the maximum oxygen demands in these batch cultures were $104 \mathrm{mmole} / \mathrm{l} . / \mathrm{hr}$. with medium $\mathrm{A}(\mu=0.78)$ and 61 with $\mathrm{B}(\mu=0 \cdot 46)$. Hence with oxygen absorption coefficients of 463 and $228 \mathrm{mmole} / \mathrm{l}$./hr., respectively, oxygen should have been present in large excess throughout the growth periods.

\section{Effect of increasing glucose concentration}

The results so far described concern the metabolism of glucose at a concentration of $1 \%(w / v)$. An investigation of the metabolism using $2 \%(w / v)$ glucose media was also made to see whether the metabolism would be as one would predict from the study of the lower strength media. A complication arose because, with the utilization of greater amounts of $\mathrm{NH}_{3}$, the steady state $\mathrm{pH}$ value (6.3) was lower than that with $1 \%$ glucose $(\mathrm{pH} 6 \cdot 7)$; at the moment one cannot say whether this affected the results. The oxygen absorption coefficient was $228 \mathrm{mmole} / \mathrm{l} / \mathrm{hr}$. The glucose utilization over the range of 
dilution rates studied was practically $100 \%$; the results are shown in Fig. 8 . The average amount of carbon accounted for as known products was $88 \%$; standard deviation 7. At dilution rates below $\mathbf{0 . 4}$ the only products found were organisms and $\mathrm{CO}_{2}$, and the number of moles of $\mathrm{CO}_{2}$ produced/mole glucose-carbon was $\mathbf{0} \cdot \mathbf{4}$, as with $1 \%$ glucose. Hence it is concluded that the metabolism is fully aerobic with $2 \%$ glucose and an oxygen absorption coefficient of $228 \mathrm{mmole} / \mathrm{l}$./hr. at dilution rates below $\mathbf{0 . 4}$. When the dilution rate

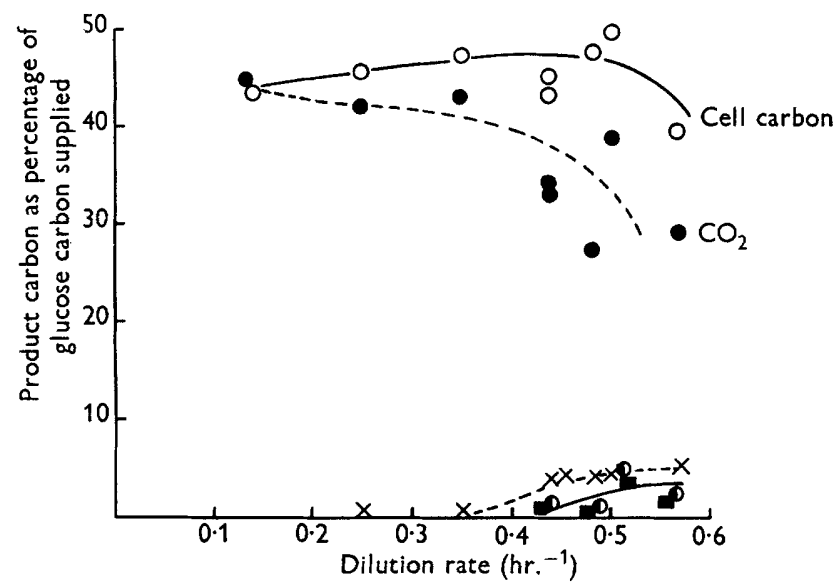

Fig. 8. Products of glucose metabolism as a function of dilution rate with a glucose supply of $19.9 \mathrm{~g} . / 1$., medium $B$ and an oxygen absorption coefficient of $228 \mathrm{mmole} / \mathrm{l} . / \mathrm{hr}$. Acetic acid, $\times$; acetoin, $\mathbf{D}$; butanediol, $\boldsymbol{D}$.

exceeded $0 \cdot 4$ acetic acid was produced and butanediol and acetoin appeared at a slightly higher dilution rate. Small amounts of formic acid and ethanol (1-2\% of the total carbon) were formed at the higher dilution rates. At dilution rates in excess of $\mathbf{0 \cdot 4}$, therefore, the metabolism was partially aerobic. The value of the oxygen demand constant calculated from the $\mathrm{CO}_{2}$ production for fully aerobic metabolism with glucose at the $2 \%$ level is close to 0.4 as it is with $1 \%$ glucose. With an oxygen absorption coefficient of $228 \mathrm{mmole} / \mathrm{l} / \mathrm{hr}$. and a glucose concentration of $19.9 \mathrm{~g}$./l. (666 mmole carbon) one calculates that the oxygen solution rate should not become a limiting factor until the dilution rate exceeds $0 \cdot 86$. There was, therefore, a considerable discrepancy between the theoretical value $(0 \cdot 86)$ and the value found $(0 \cdot 4)$ for the dilution rate at which the metabolism changed from the fully to the partially aerobic type. This discrepancy is in contrast to the much better agreement between the calculated and the values found for the dilution rate at which the change from fully aerobic to partially aerobic metabolism occurred with $1 \%$ glucose. To account for the discrepancy it was thought that the oxygen absorption coefficient might decrease with increase in the concentration of organisms as it does with moulds (Chain \& Gualandi, 1954). It was found, however, that freeze-dried Aerobacter cloacae added at a concentration of $0.8 \%(\mathrm{w} / \mathrm{v})$ to a sulphite solution decreased the oxygen absorption coefficient by only 5-10\% 
(the measurements were carried out quickly with $0 \cdot 001 \mathrm{M}-\mathrm{CuSO}_{4}$ as catalyst). Hence diffusion of oxygen from the gas to the liquid seemed to be little affected by bacteria concentrations up to $0.8 \%$ dry wt. It seems unlikely therefore that the occurrence of oxygen-limited metabolism with the $2 \%$ glucose medium and an oxygen absorption coefficient of $228 \mathrm{mmole} / \mathrm{l}$./hr. was because the oxygen solution rate was a limiting factor.

\section{DISCUSSION}

\section{Maximum oxygen uptake rate with limited aeration}

This work shows that at bacterial population densities equivalent to $c .0 \cdot 4 \%$ dry wt., when aeration becomes limiting the oxygen uptake rate is roughly equal to the maximum oxygen solution rate $(\sigma)$ as determined by the sulphite method. When, however, the population density is equivalent to $0 \cdot 8 \%$ dry wt. aeration becomes limiting when the oxygen uptake rate is considerably less than $\sigma$. This suggests that at low population densities oxygen solution rate is the sole factor limiting the availability of oxygen to the bacteria but at high population densities some other factor also is concerned. This finding is to be contrasted with that of Maxon \& Johnson (1953) who concluded that in yeast cultures with population densities up to the equivalent of $4 \%$ dry wt., aeration is never limiting until the oxygen uptake rate is equal to $\sigma$.

\section{Recognition of oxygen deficiency}

With the strain of Aerobacter cloacae used here and the $\mathrm{pH}$ value between 6 and 7 the production of acetic acid may be the first sign that there is an oxygen deficiency. With increasing oxygen deficiency 2:3-butanediol and acetoin appear in the medium and finally, as the conditions become nearly anaerobic, ethanol and formic acid appear in large amount. Decreased yields of organisms and $\mathrm{CO}_{2}$ also indicate an oxygen deficiency. Acetic acid is also produced when there is an excess of available oxygen if the organism is growing at or near to its maximum rate. This effect is analogous to one which Maxon \& Johnson (1953) observed to occur in yeast metabolism. They showed that a yeast with excess oxygen completely converted glucose to cell-carbon and $\mathrm{CO}_{2}$ provided the growth rate was not too high; however, when the yeast growth rate exceeded a certain critical value then ethanol was produced even with excess oxygen. To account for ethanol production by yeast in the presence of excess oxygen Maxon \& Johnson (1953) suggested that when a certain rate of glucose catabolism/g. organism is reached, 'the oxidative enzymes of the yeast have been saturated and the glycolytic enzymes which are present in greater abundance, operate to supply additional energy for growth'. The rate of glucose catabolism/g. organism $\left(K_{c}\right)$ for carbon-substrate-limited growth is shown at the beginning of this paper to be the quotient $\mu / \boldsymbol{Y}$. The $K_{c}$ values for $A$. cloacae under certain critical conditions are given in Table 2, from which it is clear that under conditions of oxygen deficiency the organisms can utilize glucose more rapidly than they can in the presence of oxygen. The critical $K_{c}$ 
values above which acetic acid formation occurred in the presence of excess oxygen were $48 \mathrm{mmole}$ carbon/hr./g. dry wt. when the glucose consumption was $10 \mathrm{~g}$./l. and $31 \mathrm{mmole} / \mathrm{hr}$. $/ \mathrm{g}$. dry wt. when the glucose consumption was $20 \mathrm{~g}$. 1 . The lower critical value of $K_{\mathrm{c}}$ when the glucose consumption was $20 \mathrm{~g}$./l. suggests that the occurrence of partially aerobic metabolism in that case was not due to 'saturation' of the enzymic pathway to free oxygen.

Table 2. Specific glucose-carbon catabolic rates of Aerobacter cloacae under some critical conditions

\begin{tabular}{lcc}
\multicolumn{1}{c}{ Conditions* } & $\begin{array}{c}\text { constant } \\
\left(\mathrm{hr} .^{-1}\right)\end{array}$ & $\begin{array}{c}\text { dry wt. } \\
\text { organism }\end{array}$ \\
$\begin{array}{l}\text { With excess oxygen at growth rate at which acetic acid } \\
\text { production began }\end{array}$ & 0.60 & 48 \\
At maximum growth rate with excess oxygen & 0.72 & 65 \\
$\begin{array}{l}\text { Partially aerobic metabolism }(\sigma, 24) \text {, close to maximum } \\
\text { growth rate }\end{array}$ & 0.50 & 91 \\
Anaerobic metabolism close to maximum growth rate & 0.25 & 105 \\
$\quad$ These results were obtained using medium B with 10.6 g. glucose $/$ l.
\end{tabular}

\section{Prevention of oxygen deficiency}

To show that aeration is not a limiting factor in an aerobic process it is not sufficient to demonstrate that the process is unaffected by increasing the rate of air flow through the culture as some recent papers suggest. Increasing the air flow rate does not necessarily increase the oxygen solution rate and consequently the amount of available oxygen. To demonstrate an increase in oxygen available to the culture one must demonstrate that the oxygen absorption coefficient is increased. Once the oxygen absorption coefficient is fixed, to ensure that an excess of oxygen is available to the organisms the oxygen demand must be made less than the oxygen absorption coefficient. From equation (6) it follows that the oxygen demand can be decreased either by lowering the growth-rate constant or the amount of substrate supplied. For example, applying the results to shake-flask culture in which the oxygen absorption coefficient is $40 \mathrm{mmole} / \mathrm{l}$./ hr. (a fairly high value for shake-flasks; see, for example, Finn, 1954) we find that when the generation time of the organism is one hour (i.e. $\mu=0.693$ ) and $P$ is 0.4 the glucose concentration must not exceed $4 \cdot 33 \mathrm{~g}$./l. if excess oxygen is required in the medium throughout the growth of the culture.

I wish to thank Dr D. Herbert for helpful suggestions, Mr J. Stratton for his technical assistance and Mr W. Gillett who carried out some of the analytical work. 


\section{REFERENCES}

Altermatt, H. A., Simpson, F. J. \& Neish, A. C. (1955). The fermentation of D-allose and D-glucose by Aerobacter aerogenes. Canad. J. Microbiol. 1, 473.

Bartholomew, W. M., Karow, E. O., Sfat, M. R. \& Wilhelm, R. H. (1950). Oxygen transfer and agitation in submerged fermentation. Mass transfer of oxygen in submerged fermentation of Streptomyces griseus. Industr. Engng Chem. 42, 1801.

Chain, E. B. \& Gualandi, G. (1954). Aeration studies. R.C. Ist. sup. Sanit. (English ed.), 17, 5.

Clifton, C. E. (1946). Microbial assimilations. Advanc. Enzymol. 6, 269.

Conway, E. J. (1947). Microdiffusion Analysis and Volumetric Error, London: Crosby Lockwood.

Cooper, C. M., Fernstrom, G. A. \& Miller, S. A. (1944). Performance of agitated gas-liquid contractors. Industr. Engng Chem. 36, 504.

Elsworth, R., Meakin, L. R. P., Pirt, S. J. \& Capelu, G. H. (1956). A two litre scale continuous culture apparatus for micro-organisms. J. appl. Bact. 19, 264.

FinN, R. K. (1954). Agitation-aeration in the laboratory and in industry. Bact. Rev. $18,254$.

Fujita, A. \& Iwatake, D. (1931). Determination of the true blood sugar without yeast. Biochem. $Z$. 242, 43.

HAPPOLD, F. C. \& SPENCER, C. P. (1952). The bacterial formation of acetylmethylcarbinol and 2:3-butylene glycol. Biochim. biophys. Acta, 8, 18.

Herbert, D., Elsworth, R. \& Telling, R. C. (1956). The continuous culture of bacteria; a theoretical and experimental study. J. gen. Microbiol. 14, 601.

Hixson, A. W. \& Gaden, E. L. (1950). Oxygen transfer in submerged fermentation. Industr. Engng Chem. 42, 1792.

Ledingham, G. A. \& Neish, A. C. (1954). Fermentative production of 2, 3-butanediol. Industrial Fermentations. New York: Chemical Publishing Co.

LoNGMUIR, I. S. (1954). Respiration rate of bacteria as a function of oxygen concentration. Biochem. J. 57, 81.

Maxon, W. D. \& Jonnson, M. J. (1953). Aeration studies on propagation of baker's yeast. Industr. Engng Chem. 45, 2554.

Movod, J. (1942). Recherches sur la croissance des cultures bactériennes. Paris: Hermann et Cie.

Monod, J. (1950). La technique de culture continue: théorie et applications. Ann. Inst. Pasteur, 79, 390.

Neish, A. C. (1952). Analytical Methods for Bacterial Fermentations. Saskatoon: National Research Council, Canada.

Oginsky, E. L. \& Umbreit, W. W. (1954). An Introduction to Bacterial Physiology, p. 220. San Francisco: Freeman and Co.

Prrt, S. J. (1956). The influence of oxygen on the metabolism of growing organisms determined by means of the continuous culture technique. J. gen. Microbiol. 15, iv.

Stephenson, M. (1950). Bacterial Metabolism, 3rd ed. p. 99. London: Longmans, Green and Co.

Winzler, R. J. (1941). The respiration of baker's yeast at low oxygen tension. J. cell comp. Physiol. 17, 263.

Wrse, W. S. (1950). The aeration of culture media: a comparison of the sulphite and polarographic methods. J. Soc. chem. Ind., Lond. suppl. no. 1, S. 40. 